RASĀYAN J. Chem.

Vol. 13 | No. 4 |2249-2255| October - December | 2020 ISSN: 0974-1496 | e-ISSN: 0976-0083 | CODEN: RJCABP

RJC http://www.rasayanjournal.com http://www.rasayanjournal.co.in

\title{
DETERMINATION OF BIOLOGICAL ACTIVITY, ANTIOXIDANT ACTIVITY AND SYNTHESIS OF TRIAZOLOPYRIMIDINES
}

\author{
V. P. Gilava ${ }^{1, *}$, P. K. Patel ${ }^{1}$, H. K. Ram ${ }^{2}$ and J. H. Chauhan ${ }^{2}$ \\ ${ }^{1}$ Department of Chemistry, Smt. J. A. Patel Mahila College, Morbi-363641, (Gujarat), India \\ ${ }^{2}$ Department of Chemistry, Tolani College of Arts \& Science, Adipur-370205, (Gujarat) India \\ *E-mail: ram.haresh2007@gmail.com
}

\begin{abstract}
Potent Synthesis of an uncommon series of 7-(4-(benzyloxy)-3-methoxyphenyl)-N-(4- chlorophenyl)-4,7-dihydro-5methyl-[1,2,4]triazolo[1,5-a]pyrimidine-6-carboxamide (V1-V10)was accomplished using Biginelli protocol from 4-(benzyloxy)-3-methoxybenzaldehyde, $\mathrm{N}$-(substituted phenyl)-3-oxobutanamide and 2H-1,2,4-triazol-3-amine and few drop of N,N-Dimethylformamide. All the synthesized compounds were characterized by IR, NMR, mass spectroscopic techniques and elemental analyses. All the synthesized compounds were evaluated for their antimicrobial activity and antioxidant activity.
\end{abstract}

Keywords: 4-(benzyloxy)-3-methoxybenzaldehyde, N-(substitutedphenyl)-3-oxobutanamide, 2H-1,2,4-triazol-3amine and N,N'-Dimethylformamide.

(C) RASĀYAN. All rights reserved

\section{INTRODUCTION}

Pyrimidine is a heteroatom-containing compound like pyridine. ${ }^{1}$ Pyramidine is a six-member heterocyclic ring with the nitrogen atom at 1 and 3 positions. ${ }^{2}$ This ring system has wide-spread occurrence and it is found in many biological complexes like nucleuotides, vitamin B1 and alloxane etc. ${ }^{3}$ Uric acid and Alloxane which are derivative of Pyrimidine were identified in the year 1818. Until 1876, the synthesis of pyrimidine was not reported in the literature. ${ }^{4}$ Grimauxfirst reported the work of preparation of barbituric acid from diaminomethanal and propanedioic acid with $\mathrm{POCl}_{3}$. Further, Pinner started a systematic study of pyrimidine by synthesizing pyrimidine derivatives by condensation of acetoacetate and amidines ${ }^{5,6}$. Pinner used the name 'pyrimidine' for the first time in the year $1885 .{ }^{7,8}$ Biginelli reaction is a multicomponent one-pot protocol developed by Pietro Biginelli. ${ }^{9-11}$ The reaction involves the use of catalysts like Bronsted acid or Lewis acids such as copper trichloro acetate hydrate and boron trifluoride $\left(\mathrm{BF}_{3}\right)$ and many others. ${ }^{12}$ Dihydropyrimidines and 1,2,3,4-tetrahydro- derivatives are reported to possess a wide range of biological activity. ${ }^{13-14}$ They are widely useful in various branches of Chemistry like Bioinorganic Chemistry, Medicinal Chemistry due to their biological activities. anti-microbial, antiviral, anti-carcinogenic activities etc. ${ }^{15-18}$ Some derivatives are also reported as antihypertensive agents, calcium channel blockers and alpha-antagonists. Literature reports various modifications of Biginelli reaction to get diversely substituted pyrimidine derivatives. ${ }^{19-23}$ The present work reports the preparation of multifunctionalized triazolo[ $[1,5-a]$ pyrimidines using Biginelli protocol and their antimicrobial and antioxidant activity evaluation.

\section{EXPERIMENTAL}

General Procedure for the Synthesis of Triazolo[1,5- $a$ ]pyrimidines (V1-V10)

To a mixture of 2H-1,2,4-triazol-3-amine (0.01 mol), 4-(benzyloxy)-3-methoxybenzaldehyde $(0.01 \mathrm{~mol})$, $\mathrm{N}$-(substituted phenyl)-3-oxobutanamide $(0.01 \mathrm{~mol})$ in the solvent $1-2 \mathrm{~mL}$ of DMF and few drops of con. $\mathrm{HCl}$ was added and the resulting mixture was heated for $5 \mathrm{hrs}$ in refluxing condition. The reaction mixture was kept at room temperature for $20 \mathrm{hrs}$. The thus separated crystalline product was filtered and washed with methanol.

Rasayan J. Chem., 13(4), 2249-2255(2020)

http://dx.doi.org/10.31788/ RJC.2020.1346072

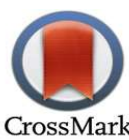


RASĀYAN J. Chem.

Vol. 13 | No. 4 |2249-2255| October - December | 2020

7-(4-(benzyloxy)-3-methoxyphenyl)-N-(4-chlorophenyl)-4,7-dihydro-5-methyl-[1,2,4]triazolo[1,5a]pyrimidine-6-carboxamide (V1)

M.P. $188^{\circ}$ C; Yield: 56\% IR (KBr) v, $\mathrm{cm}^{-1}$ : 3149, $2872(\mathrm{C}-\mathrm{H}), 3412(\mathrm{~N}-\mathrm{H}), 1676(\mathrm{C}=\mathrm{O}), 1514$ (Aromatic skeletons), 1236 (C-O-C), $773(\mathrm{C}-\mathrm{Cl}) ;{ }^{1} \mathrm{H}$ NMR (400 MHz, DMSO) : $\delta_{\mathrm{H}} 2.48$ (singlet, $3 \mathrm{H}$, methyl), 3.64 (singlet, $3 \mathrm{H}$, methoxy), 4.99 (singlet, $2 \mathrm{H},-\mathrm{CH}_{2}-\mathrm{O}$ ), 6.47 (singlet, $1 \mathrm{H},-\mathrm{CH}$ - of pyrimidine ring), 6.81-7.63 (multiplet, $13 \mathrm{H}$, Aromatic -H), 9.83 (singlet, $1 \mathrm{H}, \mathrm{NH}-\mathrm{CO}$ ), 10.22 (singlet, $1 \mathrm{H},-\mathrm{NH}$ - Aromatic-NH); MS: $m / z$ 501.Anal.found: $\mathrm{C}, 64.60 ; \mathrm{H}, 4.82 ; \mathrm{Cl}, 7.06 ; \mathrm{N}, 13.95 . \mathrm{C}_{27} \mathrm{H}_{24} \mathrm{ClN}_{5} \mathrm{O}_{3}$ requires: $\mathrm{C}, 64.65 ; \mathrm{H}, 4.82 ; \mathrm{Cl}$, $7.01 ; \mathrm{N}, 13.92 \%$.

7-(4-(benzyloxy)-3-methoxyphenyl)-N-(3-chlorophenyl)-4,7-dihydro-5-methyl-[1,2,4]triazolo[1,5a]pyrimidine-6-carboxamide (V2)

M.P. $180^{\circ}$ C; Yield: $60 \%$; IR( $\left.\mathrm{KBr}\right) \mathrm{vcm}^{-1}$ : 3133, $2870(\mathrm{C}-\mathrm{H}), 3423(\mathrm{~N}-\mathrm{H}), 1673(\mathrm{C}=\mathrm{O}), 1501$ (Aromatic skeletons), 1229 (C-O-C), $782(\mathrm{C}-\mathrm{Cl}) ;{ }^{1} \mathrm{H}$ NMR (400 MHz, DMSO) : $\delta_{\mathrm{H}} 2.51$ (singlet, $3 \mathrm{H}$, methyl), 3.61 (singlet, $3 \mathrm{H}$, methoxy), 5.10 (singlet, $2 \mathrm{H},-\mathrm{CH}_{2}-\mathrm{O}$ ), 6.41 (singlet, $1 \mathrm{H}$, -CH- of pyrimidine ring), 6.77-7.76 (multiplet, 13H, Aromatic -H) 9.74 (singlet, 1H, -NH-CO-), 10.10 (singlet, 1H, -NH- Aromatic-NH); MS: $m / z$ 501.Anal.found: $\mathrm{C}, 64.60 ; \mathrm{H}, 4.82 ; \mathrm{Cl}, 7.06 ; \mathrm{N}, 13.95 . \mathrm{C}_{27} \mathrm{H}_{24} \mathrm{ClN}_{5} \mathrm{O}_{3}$ requires: $\mathrm{C}, 64.61 ; \mathrm{H}, 4.80$; $\mathrm{Cl}, 7.07 ; \mathrm{N}, 13.88 \%$.

\section{7-(4-(benzyloxy)-3-methoxyphenyl)-N-(2-chlorophenyl)-4,7-dihydro-5-methyl-[1,2,4]triazolo[1,5-} a]pyrimidine-6-carboxamide (V3)

M.P. $171^{\circ} \mathrm{C}$; Yield: 57\%; IR(KBr) v cm ${ }^{-1}: 3173,2876(\mathrm{C}-\mathrm{H}), 3349(\mathrm{~N}-\mathrm{H}), 1670(\mathrm{C}=\mathrm{O}), 1539,1514$ (Aromatic skeletons), 1229 (C-O-C), $689(\mathrm{C}-\mathrm{Cl}) ;{ }^{1} \mathrm{H}$ NMR $(400 \mathrm{MHz}, \mathrm{DMSO}): \delta_{\mathrm{H}} 2.55$ (singlet, $3 \mathrm{H}$, $\mathrm{CH}_{3}$ ), 3.49 (singlet, $3 \mathrm{H}, \mathrm{OCH}_{3}$ ), 4.97 (singlet, $2 \mathrm{H},-\mathrm{CH}_{2}-\mathrm{O}$ ), 6.38 (singlet, $1 \mathrm{H},-\mathrm{CH}$ - of pyrimidine ring), 7.18-7.92 (multiplet, 13H, Aromatic-H), 9.35 (singlet, 1H, -NH-CO-), 9.97 (singlet, 1H, -NHAromatic-NH-); MS: $m / z$ 501.Anal.found: $\mathrm{C}, 64.60 ; \mathrm{H}, 4.82 ; \mathrm{Cl}, 7.06 ; \mathrm{N}, 13.95 . \mathrm{C}_{27} \mathrm{H}_{24} \mathrm{ClN}_{5} \mathrm{O}_{3}$ requires: C, 64.58; H, 4.71; Cl, 7.14; N, 13.75\%.

7-(4-(benzyloxy)-3-methoxyphenyl)-N-(4-fluorophenyl)-4,7-dihydro-5-methyl-[1,2,4]triazolo[1,5a]pyrimidine-6-carboxamide (V4)

M.P. $169^{\circ} \mathrm{C}$; Yield: 65\%;IR(KBr) v cm ${ }^{-1}: 3173,2868(\mathrm{C}-\mathrm{H}), 3356(\mathrm{~N}-\mathrm{H}), 1678(\mathrm{C}=\mathrm{O})$, 1556,1523 (Aromatic skeletons), 1223 (C-O-C), 1043 (C-F); ${ }^{1} \mathrm{H}$ NMR (400 MHz, DMSO) : $\delta_{\mathrm{H}} 2.51$ (singlet, $3 \mathrm{H}$, methyl), 3.40 (singlet, $3 \mathrm{H}$, methoxy), 4.84 (singlet, $2 \mathrm{H},-\mathrm{CH}_{2}-\mathrm{O}$ ), 6.45 (singlet, $1 \mathrm{H}$, - $\mathrm{CH}$ - of pyrimidine), 7.73-8.35 (multiplet, 13H, Aromatic-H), 9.42 (singlet, 1H, -NH-CO-), 9.90 (singlet, 1H, -NHAromatic-NH); MS: $m / z$ 485.Anal.found: $\mathrm{C}, 66.79 ; \mathrm{H}, 4.98 ; \mathrm{F}, 3.91 ; \mathrm{N}, 14.42 . \mathrm{C}_{27} \mathrm{H}_{24} \mathrm{FN}_{5} \mathrm{O}_{3}$ requires: $\mathrm{C}$, $66.70 ; \mathrm{H}, 4.91 ; \mathrm{F}, 4.05 ; \mathrm{N}, 14.25 \%$.

\section{7-(4-(benzyloxy)-3-methoxyphenyl)-N-(3-fluorophenyl)-4,7-dihydro-5-methyl-[1,2,4]triazolo[1,5-} a]pyrimidine-6-carboxamide(V5)

M.P. $164^{\circ} \mathrm{C}$; Yield: 51\%; IR(KBr) v cm ${ }^{-1}: 3133,2852(\mathrm{C}-\mathrm{H}), 3278(\mathrm{~N}-\mathrm{H}), 1678(\mathrm{C}=\mathrm{O}), 1563,1513$ (Aromatic skeletons), 1219 (C-O-C), 1049 (C-F); ${ }^{1} \mathrm{H}$ NMR (400 MHz, DMSO) : $\delta_{\mathrm{H}} 2.43$ (singlet, 3H, methyl), 3.64 (singlet, $3 \mathrm{H}$, methoxy), 4.44 (singlet, $2 \mathrm{H},-\mathrm{CH}_{2}-\mathrm{O}$ ), 6.64 (singlet, $1 \mathrm{H}$, $-\mathrm{CH}$ - of pyrimidine), 7.70-8.31 (multiplet, 13H, Aromatic-H), 9.51 (singlet, 1H, -NHCO-), 9.89 (singlet, $1 \mathrm{H}$, -NH- AromaticNH-); MS: $m / z$ 485.Anal.found: $\mathrm{C}, 64.60 ; \mathrm{H}, 4.82 ; \mathrm{Cl}, 7.08 ; \mathrm{N}, 13.91 . \mathrm{C}_{27} \mathrm{H}_{24} \mathrm{FN}_{5} \mathrm{O}_{3}$ requires: $\mathrm{C}, 64.37 ; \mathrm{H}$, $4.06 ; \mathrm{F}, 8.14 ; \mathrm{N}, 13.45 \%$.

7-(4-(benzyloxy)-3-methoxyphenyl)-N-(2-fluorophenyl)-4,7-dihydro-5-methyl-[1,2,4]triazolo[1,5a]pyrimidine-6-carboxamide(V6)

M.P. $170^{\circ} \mathrm{C}$; Yield: 53\%; IR(KBr) v cm$~_{-1}$ : 3109, $2862(\mathrm{C}-\mathrm{H}), 3313(\mathrm{~N}-\mathrm{H}), 1671(\mathrm{C}=\mathrm{O}), 1562,1524$ (Aromatic skeletons), 1213 (C-O-C), 1060 (C-F); ${ }^{1} \mathrm{H}$ NMR (400 MHz, DMSO) : $\delta_{\mathrm{H}} 2.47$ (singlet, $3 \mathrm{H}$, methyl), 3.54 (singlet, $3 \mathrm{H}$, methoxy), 4.40 (singlet, $2 \mathrm{H},-\mathrm{CH}_{2}-\mathrm{O}$ ), 6.49 (singlet, $1 \mathrm{H},-\mathrm{CH}$ - of pyrimidine ring), 7.46-8.10 (multiplet, 13H, Aromatic-H) 9.46 (singlet, 1H, -NH-CO-), 9.79 (singlet, 1H, -NHAromatic-NH-); MS: $m / z$ 485.Anal.found: C, 64.60; $\mathrm{H}, 4.82 ; \mathrm{Cl}, 7.06 ; \mathrm{N}, 13.95 . \mathrm{C}_{27} \mathrm{H}_{24} \mathrm{FN}_{5} \mathrm{O}_{3}$ requires: $\mathrm{C}$, $64.39 ; \mathrm{H}, 4.02 ; \mathrm{F}, 8.11 ; \mathrm{N}, 13.40 \%$. 
RASĀYAN J. Chem.

Vol. 13 | No. 4 |2249-2255| October - December | 2020

7-(4-(benzyloxy)-3-methoxyphenyl)-N-(4-bromophenyl)-4,7-dihydro-5-methyl-[1,2,4]triazolo[1,5a]pyrimidine-6-carboxamide (V7)

M.P. $176^{\circ} \mathrm{C}$; Yield: 57\%; IR(KBr) v cm ${ }^{-1}: 3115,2876(\mathrm{C}-\mathrm{H}), 3334(\mathrm{~N}-\mathrm{H}), 1668(\mathrm{C}=\mathrm{O})$, 1577, 1517 (Aromatic skeletons), 1209 (C-O-C), $650(\mathrm{C}-\mathrm{Br}) ;{ }^{1} \mathrm{H}$ NMR $(400 \mathrm{MHz}, \mathrm{DMSO}): \delta_{\mathrm{H}} 2.34$ (singlet, $3 \mathrm{H}$, methyl), 3.49 (singlet, $3 \mathrm{H}$, methoxy), 4.41 (singlet, $2 \mathrm{H},-\mathrm{CH}_{2}-\mathrm{O}$ ), 6.12 (singlet, $1 \mathrm{H}$, $-\mathrm{CH}$ - of pyrimidine ring), 7.33-8.69 (multipletm, 13H, Aromatic -H-), 9.69 (singlet, 1H, -NHCO-), 10.19 (singlet, 1H, -NHAromatic -NH-); MS: $m / z$ 546.Anal.found: C, $59.35 ; \mathrm{H}, 4.43 ; \mathrm{Br}, 14.62 ; \mathrm{N}, 12.82 . \mathrm{C}_{27} \mathrm{H}_{24} \mathrm{BrN}_{5} \mathrm{O}_{3}$ requires: C, 59.13; H, 4.69; Br, 14.23; N, 12.74\%.

\section{7-(4-(benzyloxy)-3-methoxyphenyl)-N-(3-bromophenyl)-4,7-dihydro-5-methyl-[1,2,4]triazolo[1,5- a]pyrimidine-6-carboxamide (V8)}

M.P. $172^{\circ}$ C; Yield: 58\%; IR(KBr) v cm ${ }^{-1}: 3109,2870(\mathrm{C}-\mathrm{H}), 3319(\mathrm{~N}-\mathrm{H}), 1659(\mathrm{C}=\mathrm{O}), 1564,1509$ (Aromatic skeletons), 1218 (C-O-C), $642(\mathrm{C}-\mathrm{Br}) ;{ }^{1} \mathrm{H}$ NMR (400 MHz, DMSO) : $\delta \mathrm{H} 2.29$ (singlet s, 3H, methyl), 3.57 (singlet, $3 \mathrm{H}$, methoxy), 4.36 (singlet s, $2 \mathrm{H},-\mathrm{CH}_{2}-\mathrm{O}$ ), 6.48 (singlet, $1 \mathrm{H}$, - $\mathrm{CH}$ - of pyrimidine ring), 7.41-8.47 (multiplet, 13H, Aromatic -H-), 9.71 (singlet s, 1H, -NHCO-), 10.04 (singlet, 1H, -NHAromatic -NH-); MS: $m / z$ 546.Anal.found: C, 59.35; H, 4.43; Br, 14.62; N, 12.82. $\mathrm{C}_{27} \mathrm{H}_{24} \mathrm{BrN}_{5} \mathrm{O}_{3}$ requires: C, 59.11; H, 4.64; Br, 14.27; N, $12.71 \%$.

\section{7-(4-(benzyloxy)-3-methoxyphenyl)-N-(2-bromophenyl)-4,7-dihydro-5-methyl-[1,2,4]triazolo[1,5- a]pyrimidine-6-carboxamide (V9)}

M.P. $163^{\circ} \mathrm{C}$; Yield: 52\%; IR(KBr) v cm ${ }^{-1}: 3170,2861(\mathrm{C}-\mathrm{H}), 3324(\mathrm{~N}-\mathrm{H}), 1645(\mathrm{C}=\mathrm{O}), 1565,1507$ (Aromatic skeletons), 1208 (C-O-C), $634(\mathrm{C}-\mathrm{Br}) ;{ }^{1} \mathrm{H}$ NMR $(400 \mathrm{MHz}, \mathrm{DMSO}): \delta_{\mathrm{H}} 2.46$ (singlet, $3 \mathrm{H}$, methyl), 3.38 (singlet, $3 \mathrm{H}$, methoxy), 4.52 (singlet, $2 \mathrm{H},-\mathrm{CH}_{2}-\mathrm{O}$ ), 6.78 (singlet, $1 \mathrm{H}$, $-\mathrm{CH}$ - of pyrimidine ring), 7.61-8.51 (multiplet, 13H, Aromatic -H-), 9.72 (singlet, 1H, -NHCO-), 10.17 (singlet, 1H, -NHAromatic-NH-); MS: $m / z$ 546.Anal.found: $\mathrm{C}, 59.35 ; \mathrm{H}, 4.43 ; \mathrm{Br}, 14.62 ; \mathrm{N}, 12.82 . \mathrm{C}_{27} \mathrm{H}_{24} \mathrm{BrN}_{5} \mathrm{O}_{3}$ requires: C, 59.21; H, 4.59; Br, 14.21; N, 12.61\%.

\section{7-(4-(benzyloxy)-3-methoxyphenyl)-N-(4-methylphenyl)-4,7-dihydro-5-methyl-[1,2,4] triazolo[1,5- a]pyrimidine-6-carboxamide(V10)}

M.P. $158^{\circ} \mathrm{C}$; Yield: 56\%; IR(KBr) v cm ${ }^{-1}: 3163,2853(\mathrm{C}-\mathrm{H}), 3300(\mathrm{~N}-\mathrm{H}), 1657(\mathrm{C}=\mathrm{O}), 1571,1511$ (Aromatic skeletons), 1213 (C-O-C); ${ }^{1} \mathrm{H}$ NMR (400 MHz, DMSO) : $\delta_{\mathrm{H}} 2.13$ (singlet, 3H, methyl), 2.43 (singlet, $3 \mathrm{H}$, methyl), 3.46 (singlet, $3 \mathrm{H}$, methoxy), 4.49 (singlet, $2 \mathrm{H},-\mathrm{CH}_{2}-\mathrm{O}$ ), 6.64 (singlet, $1 \mathrm{H},-\mathrm{CH}$ - of pyrimidine), 7.54-8.13 (multiplet, 13H, Aromatic -H-), 9.84 (singlet, $1 \mathrm{H},-\mathrm{NHCO}-$ ), 10.10 (singlet, 1H, NH- Aromatic-NH-); MS: $m / z$ 481.Anal.found: $\mathrm{C}, 69.84 ; \mathrm{H}, 5.65 ; \mathrm{N}, 14.54 . \mathrm{C}_{28} \mathrm{H}_{27} \mathrm{~N}_{5} \mathrm{O}_{3}$ requires: $\mathrm{C}$, $69.81 ; \mathrm{H}, 5.67$; N, $14.51 \%$.

\section{Antimicrobial Activity}

The 'in vitro' antimicrobial evaluation was carried out against standard strains of Gram +ve and Gram -ve bacteria and fungi. The standard strains were procured from the Microbial Type Culture Collection (MTCC), Gene Bank, Institute of Microbial Technology, Chandigarh, India. The determination of antimicrobial activity was done by the broth dilution method. Determination of antimicrobial activity was done against two-gram positive bacterial strains, (Staphylococcus aureus MTCC-96, Streptococcus pyogenes MTCC 443), two Gram-negative bacterial strains (Escherichia coli MTCC 442, Pseudomonas aeruginosa MTCC 441) and three fungal strains (Candidaalbicans MTCC 227, Aspergillus Niger MTCC 282, Aspergillusclavatus MTCC 1323) by minimum inhibitory concentration (MIC) method. Here Ampicillin, Nystatin Chloramphenicol, Griseofulvinand Norfloxacin were used as standard drugs. The minimal inhibitory concentration (MIC) values determined in vitro by broth dilution method for all the compounds V1-V10, specified as the minimum concentration of the compound to stop the detectable growth of specific microorganism. Serial dilutions of the test compounds and reference drugs were prepared in Muellere-Hinton agar. Drugs $(10 \mathrm{mg}$ ) were dissolved in dimethyl sulfoxide (DMSO, $1 \mathrm{~mL}$ ). Further progressive dilutions with melted Muellere-Hinton agar were prepared to obtain the desired concentrations. In primary screening $1000 \mu \mathrm{g} \mathrm{mL}^{-1}, 500 \mu \mathrm{g} \mathrm{mL}^{-1}$, and $250 \mu \mathrm{g} \mathrm{mL}^{-1}$ concentrations of the 
RASĀYAN J. Chem.

Vol. 13 | No. 4 |2249-2255| October - December | 2020

synthesized drugs were taken. The active synthesized drugs found in this primary screening were further tested in the second set of dilution at $125 \mu \mathrm{g} \mathrm{mL}^{-1}, 62.5 \mu \mathrm{g} \mathrm{mL}^{-1}, 50 \mu \mathrm{g} \mathrm{mL}^{-1}, 25 \mu \mathrm{g} \mathrm{mL}^{-1}, 12.5 \mu \mathrm{g} \mathrm{mL}^{-1}$, and $6.250 \mu \mathrm{g} \mathrm{mL} \mathrm{m}^{-1}$ concentration against all microorganisms. The tubes were inoculated with $10^{8} \mathrm{cfu} \mathrm{\textrm {mL } ^ { - 1 }}$ (colony-forming unit $/ \mathrm{mL}$ ) and incubated at $37^{\circ} \mathrm{C}$ for $24 \mathrm{~h}$. The MIC was the lowest concentration of the tested compound that yields no visible growth (turbidity) on the plate. To ensure that the solvent is inert on the bacterial growth, a control was tested with the test medium supplemented with DMSO at the same dilutions as used in the experiments and it was observed that DMSO had no effect on the microorganisms in the concentrations studied. The results obtained from antimicrobial susceptibility testing are depicted in Table-1 and Table-2.

\section{Antioxidant Activity}

Antioxidant activity of thetriazolopyrimidines (V1-V10) was determined by ABTS (2,2-diphenyle 1picrylhydrazyl) free radical assay based on the scavenging effect of them. The results are depicted in Table3.

\section{RESULTS AND DISCUSSION}

Our investigation was focused on the preparation of diversely functionalized triazolo[1,5-a]pyrimidines via a suitable and facile method.<smiles>COc1cc(C=O)ccc1OCc1ccccc1COc1ccc(NC(=O)C2=C(C)Nc3ncnn3C2c2ccc(I)c(NC(=O)C(C)=O)c2)cc1OCc1ccccc1</smiles>

$\mathrm{R}=\mathrm{Cl}, \mathrm{Br}, \ldots \ldots$

Scheme-1: Reagents and Conditions: 2H-1,2,4-triazol-3-amine, 4-(benzyloxy)-3-methoxybenzaldehyde and N(substitutedphenyl)-3-oxobutanamide, DMF, reflux, $5 \mathrm{hrs}$.

\section{Antimicrobial Activity}

The minimum inhibitory concentration (MIC) values measured in antibacterial and antifunagal activity studies of the compounds are given in the following Table-1.

Table-1: In vitro Antibacterial Screening Results for (V1-V10)

\begin{tabular}{c|c|c|c|c}
\hline Compound & \multicolumn{3}{|c}{ Minimal Inhibition Concentration $(\mu \mathrm{g}$ ml-1) } \\
\hline & \multicolumn{2}{|c|}{ Gram-positive } & \multicolumn{2}{c}{ Grame-negative } \\
\hline & S.a & S.p. & E.c. & P.a. \\
\hline V1 & 250 & 125 & 250 & 250 \\
\hline V2 & 500 & 125 & 250 & 500 \\
\hline V3 & 250 & 250 & 500 & 250 \\
\hline V4 & 500 & 250 & 500 & 250 \\
\hline V5 & 125 & 125 & 125 & 500 \\
\hline V6 & 250 & 500 & 125 & 500 \\
\hline V7 & 500 & 250 & 125 & 250 \\
\hline V8 & 500 & 250 & 250 & 125 \\
\hline V9 & 250 & 250 & 250 & 125 \\
\hline V10 & 250 & 250 & 250 & 250 \\
\hline Ampicillin & 250 & 100 & 100 & 100 \\
\hline Chloramphenicol & 50 & 50 & 50 & 50 \\
\hline Norfloxacin & 10 & 10 & 10 & 10 \\
\hline & & & &
\end{tabular}

2252 
RASĀYAN J. Chem.

Vol. 13 | No. 4 |2249-2255| October - December | 2020

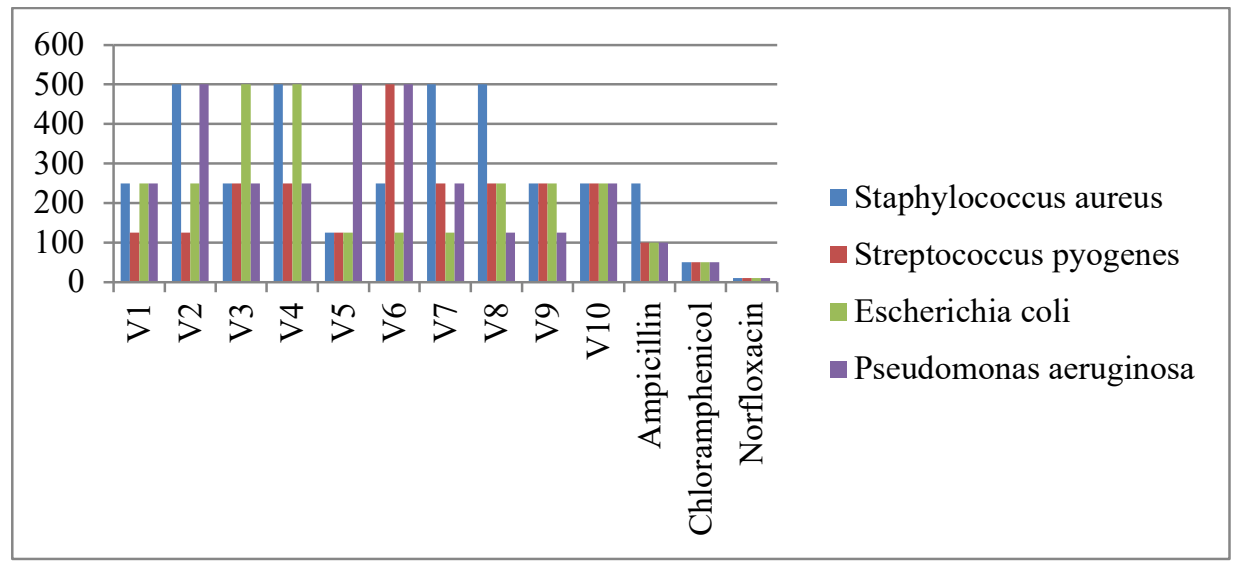

Fig.-1: Antibacterial Activity of Triazolopyrimidines V1-V10

The biological screening revealed that compound V5 exhibited maximum antibacterial activity against gram-positive bacteria and E. coli. at $125 \mu \mathrm{g} \mathrm{mL} \mathrm{m}^{-1}$. Compounds V2 and V4 showed minimum antibacterial activity. Rest of the compounds were found to be good to moderate inhibitors against tested microbial strains.

Table-2: In vitro Anti-Fungal Activity Screening Results for (V1-V10)

\begin{tabular}{c|c|c|c}
\hline \multirow{2}{*}{ Compound } & \multicolumn{3}{|c}{$\begin{array}{c}\text { Minimal Inhibition } \\
\text { Concentration }(\mu \mathrm{g} \text { ml-1) }\end{array}$} \\
\hline & \multicolumn{3}{|c}{ Fungal Species } \\
\hline & C.a. & A.n. & A.c. \\
\hline V1 & 250 & 125 & 500 \\
\hline V2 & 250 & 500 & 125 \\
\hline V3 & 350 & 125 & 125 \\
\hline V4 & 250 & 500 & 125 \\
\hline V5 & 500 & 125 & 250 \\
\hline V6 & 250 & 500 & 125 \\
\hline V7 & 500 & 125 & 125 \\
\hline V8 & 250 & 500 & 250 \\
\hline V9 & 250 & 125 & 125 \\
\hline V10 & 500 & 500 & 250 \\
\hline Nystatin & 100 & 100 & 100 \\
\hline Griseofulvin & 450 & 100 & 100 \\
\hline
\end{tabular}

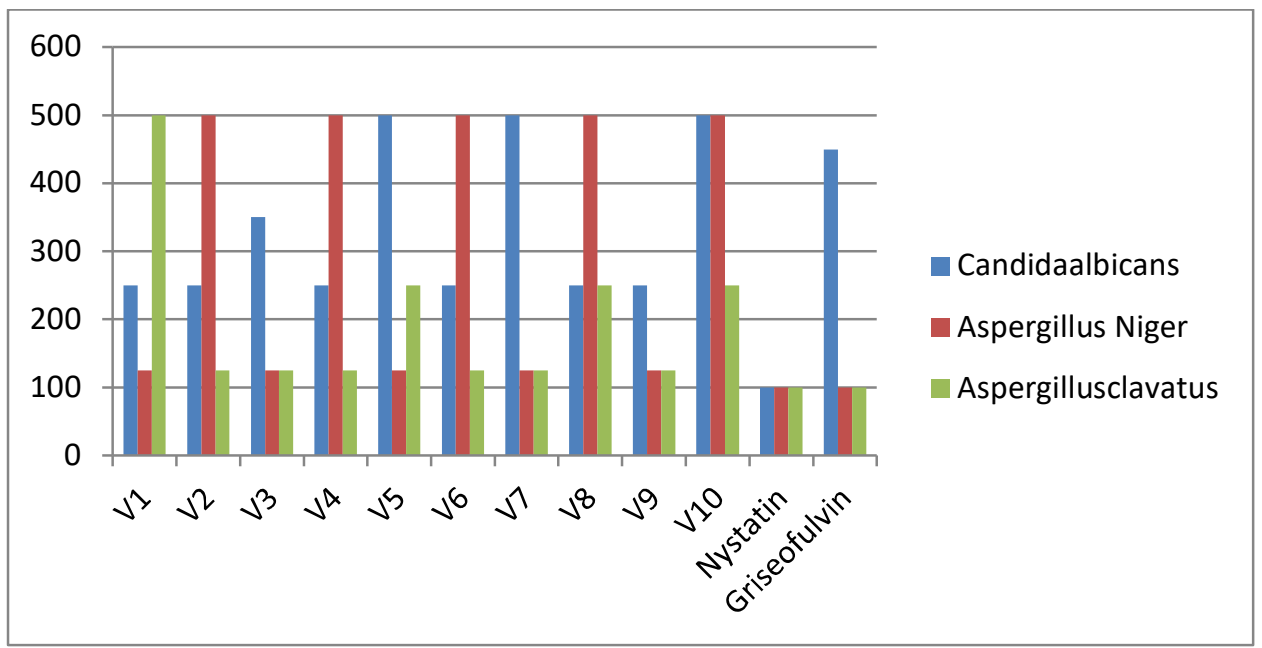

Fig.-2: Antifungal Activity of Triazolopyrimidines V1-V10 
RASĀYAN J. Chem.

Vol. 13 | No. 4 |2249-2255| October - December | 2020

\section{Antifungal Activity}

Antifungal screening of these compound results that compound V3, V7 and V9 showed good antifungal activity against Aspergillus niger and Aspergillus clavatus while remaining all compounds were good to moderate inhibitors.

\section{Antioxidant Activity}

The results of the antioxidant activity are depicted in Table-3.

Table-3: Antioxidant Activity Results for (V1-V10)

\begin{tabular}{c|c|c|c|c|c}
\hline Compound & \multicolumn{5}{|c}{ Antioxident Activity of Compounds } \\
\hline & \multicolumn{6}{|c}{ Different Concentration $\mu \mathrm{g}$ ml-1) of Compounds \% } \\
\hline & $25 \%$ & $50 \%$ & $100 \%$ & $200 \%$ & $400 \%$ \\
\hline V1 & 56.61 & 61.23 & 60.19 & 59.23 & 53.46 \\
\hline V2 & 63.31 & 58.26 & 61.01 & 58.23 & 59.13 \\
\hline V3 & 74.46 & 74.29 & 77.78 & 79.14 & 80.39 \\
\hline V4 & 64.13 & 55.13 & 53.98 & 64.21 & 64.16 \\
\hline V5 & 53.12 & 54.19 & 46.13 & 66.55 & 59.15 \\
\hline V6 & 55.19 & 57.64 & 48.59 & 66.36 & 60.18 \\
\hline V7 & 75.56 & 79.35 & 80.11 & 78.12 & 81.46 \\
\hline V8 & 58.25 & 66.36 & 66.89 & 63.25 & 65.19 \\
\hline V9 & 62.23 & 65.25 & 61.46 & 62.15 & 66.29 \\
\hline V10 & 61.16 & 66.18 & 58.49 & 58.12 & 59.36 \\
\hline Ascorbic acid & 82.73 & 87.56 & 93.17 & 94.22 & 97.51 \\
\hline
\end{tabular}

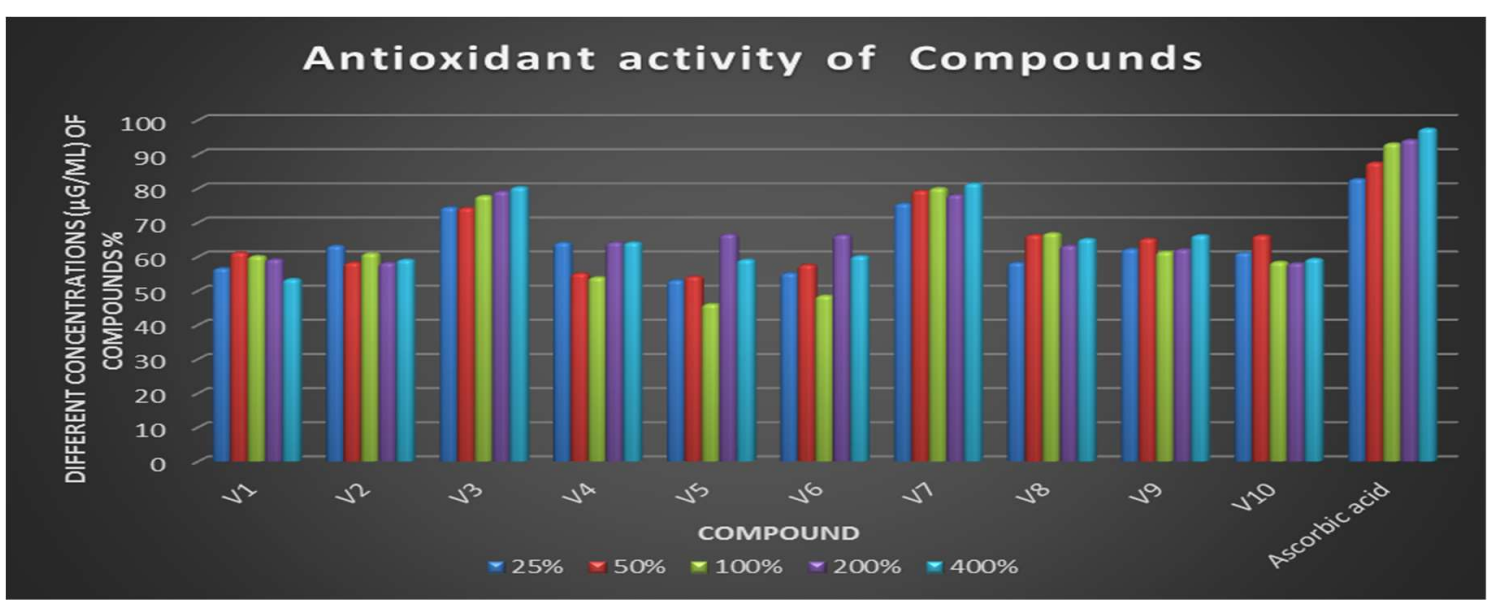

Fig.-3: Antioxidant Activity of Triazolopyrimidines V1-V10

Compounds V3 and V7 showed good antioxidant activity while the other triazolopyrimidines showed moderate to low antioxidant activity as compared to the standard Ascorbic acid.

\section{CONCLUSION}

This facile one-pot method efficiently produced diversely functionalized triazolo[1,5- $a]$ pyrimidine derivatives. The one-pot method is easy and gives the title compounds in good yield and purity. The protocol does not require tedious isolation or purification methods. The newly synthesized triazolo[1,5a]pyrimidines showed promising antibacterial, antifungal and antioxidant activities. Further structure modification and SAR studies will surely assess the biological importance of these molecules in detail.

\section{ACKNOWLEDGEMENT}

The authors are thankful to Smt. J. A. Patel Mahila College, Morbi for providing laboratory facilities.

\section{REFERENCES}

1. L. T. Gilchrist, Heterocyclic Chemistry (3th ed.),(1997).

2. A. J. Joule, and K. Mills, Heterocyclic Chemistry (5th ed.),(2010) 
RASĀYAN J. Chem.

Vol. 13 | No. 4 |2249-2255| October - December | 2020

3. M. I. Lagoja, Chemistry and Biodiversity, 2, 1(2005), DOI:10.1002/cbdv.200490173

4. E. Grimaux,Comptes Rendus Hebdomadaires des Séances de l'Académie des Sciences, 88, 85(1879).

5. G. W. Kenner, A. Todd,and R. C. Elderfield, Heterocyclic Compounds, 6, New York, Wiley, 235(1957)

6. A. Pinner, Berichte der Deutschen Chemischen Gesellschaft, A-17, 2519(1884), DOI: 10.1002/cber.18840170205

7. A. Pinner, Berichte der Deutschen Chemischen Gesellschaft, A-18, 759(1885), DOI: $10.1002 /$ cber.188501801161

8. S. Gabriel, Berichte der Deutschen Chemischen Gesellschaft, A-33, 3666(1900), DOI: $10.1002 /$ cber.190003303173

9. B. Lythgoe, and L. S. Rayner, Journal of the Chemical Society, 2323(1951), DOI: $10.1039 / \mathrm{JR} 9510002323$

10. D. J. Brown, R. F. Evans, W. B. Cowden, and M. D. Fenn, the Pyrimidines, 52, (1994), DOI: $10.1002 / 9780470187395$

11. D. Song, R. Wang, Y. Chen, S. Zhang, and C. Liu, Reaction Kinetics and Catalysis Letters, 95, 385(2008), DOI: $10.1007 / \mathrm{s} 11144-008-5379-2$

12. E. H.; Hu, D. R. Sidler, and U. H. Dolling, The Journal of Organic Chemistry, 63, 3454(1998), DOI: $10.1021 /$ jo970846u

13. P. Wipf, A. Cunningham, Tetrahedron Letters, 36,7819(1995), DOI:10.1016/0040-4039(95)01660-A

14. C.O. Kappe, Bioorganic \& Medicinal Chemistry, 10, 49(2000),DOI:10.1016/s0960-894x(99)00572-7

15. C. O. Kappe, European Journal of Medicinal Chemistry, 35,1043(2000),DOI:10.1016/s02235234(00)01189-2

16. K. S. Atwal, B. N. Swanson, S. E. Umger, D.M. Floyd, S. Moreland, A. Hedberg, and B. C. Reilly, Journal of Medicinal Chemistry, 34, 806(1991), DOI:10.1021/jm00106a048.

17. S. Rajasekaran, K. R. Gopal, P. N. Sanjay Pai, and A. Alook Kumar, International Journal of Pharm Tech Research, 3, 626(2011).

18. M. A. El- Kashouti, S. S.Tawfik, and K. A. Ahmed, S. H. Mohamed, Research Journal of Chemical Sciences, 2(9), 79(2012).

19. P. Biginelli, Gazz. Chemica Italian, 23, 360(1893).

20. C. O. Kappe, Tetrahedron, 49, 6937(1993), DOI:10.1016/S0040-4020(01)87971-0

21. E. H. Hu,D. R. Sidler,and U. H. Dolling, the Journal of Organic Chemistry, 63, 3454(1998).

22. M. B. Wahid, K. K.Tamer, A. E. Fakhry,and R. H. Ahmed, Journal Heterocyclic Chemistry, 51, 106(2014), DOI: 10.1002/jhet.2019.

23. A. E. Amr, A. M. Mohamed, S. F. Mohamed, N. A. Abdel-Hafez, and A. G. Hammam, Bioorganic \& Medicinal Chemistry, 14, 5481(2006), DOI:10.1016/j.bmc.2006.04.045.

[RJC-6072/2020] 